\title{
A model for commercializing the outcomes of innovation/research activity in universities
}

\author{
Vladislav Bogovin, and Evgeniya Vidishcheva,* \\ Sochi State University, Plastunskaya Str., 94, 354000 Sochi, Russia
}

\begin{abstract}
Innovation activity is central to economic modernization. Despite the fact that Russia possesses serious scientific/technical potential, it has fallen significantly behind industrial countries in getting innovative solutions to an open commercial market. The nation has had difficulties in commercializing innovative output, which has kept it from achieving the economic returns that it deserves. The commercialization of R\&D outcomes is indispensable to the strategic development of any organization. The insufficiently effective realization of innovation potential in Russia is largely due to the detachedness of innovation activity in higher education institutions from practice and a lack of interest from business - a key agent of innovation commercialization. The research reported in this paper focuses on the scientific/theoretical and practical/applied foundations of the process of commercializing the outcomes of innovation activity in higher education institutions. It has helped establish and gain an insight into some of the key issues in the area. The paper makes a case for the possibility of enhancing this process via a special model for commercializing the outcomes of innovation activity in higher education institutions.
\end{abstract}

\section{Introduction}

In the present-day knowledge economy, the entrepreneurial university is viewed as a dominant force that drives innovation, creative endeavor, and economic growth. Exploring the key issues that characterize academic entrepreneurship with a focus on identifying proper areas for research and appropriate mechanisms for interacting with the market forms the basis for detailed analysis of this phenomenon and may help gain a deeper insight into the current relationship between science and business. Over the course of centuries, the university has been turning into an institution where knowledge can also be created and then put to use.

Successful innovation in the present-day economy is a complex process involving interaction and two-way feedback between academic research, industrial R\&D, marketing and customer relations. Ideally, research organizations, business and the state ought to forge stable, long-term triple relations focusing on deriving value on a consistent and reliable basis.

Advantage will be with universities and organizations that, going forward, will engage in mutually beneficial cooperation by way of joint establishments as a source of new technology, which is a major catalyst for innovation-based development [1].

\footnotetext{
* Corresponding author: evgenia-vv@mail.ru
} 
Issues related to innovation-focused entrepreneurship, innovation, interaction between researchers, the state and business, technology transfer from researchers to business, and commercialization of innovation and intellectual property have been explored by Leydesdorff (2010), Munari, Sobrero, and Toschi (2015), Gulbranson and Audretsch (2008), Link and Hayter (2015), Gokhberg, Kuznetsova, and Pikalova (2018), and many others.

\section{Materials and methods}

The purpose of the research reported in this paper was to develop a structural/logical model for the mechanism of the commercialization of innovation activity in higher education institutions with participation from science, business, and the state. This model is based on engaging in the examination of existing and emerging innovations in the early stages of the research process the so-called proof of concept center (PoCC).

The work employed several empirical and theoretical methods, including data collection, interpretation, and analysis, summarization, comparison, and classification.

\section{Results and discussion}

In recent years, national governments and regional authorities around the world have focused increasingly on the development of activities on technology transfer with the aim of easing the flow of ideas from universities into industry. Insufficient sources and low volumes of private funding to support this activity are major barriers to effective commercialization of university-generated technologies today. Conventional sources of funding for expanded production are now of limited significance for researchers and university technologies, as they are characterized by significant information asymmetry, uncertain technical and commercial success, and long-term prospects for commercialization.

In addition, there are certain barriers, like researchers' lack of entrepreneurial experience and educational institutions' lack of sustainable links with the market, that limit the transformation of research-based inventions into successful products or services [2].

This gap is largely due to the "embryonic" nature of university-generated inventions (Table 1 - TRLs 1-3), which tend to function not even as a prototype - this entails a significant amount of risk associated with their subsequent validation, industrialization, and commercialization (Table 1 - TRLs 7-9) [3].

Table 1. Technology Readiness Levels (TRLs).

\begin{tabular}{|c|c|}
\hline Stage & Description \\
\hline TRL 1 & $\begin{array}{c}\text { Basic research: initial scientific research begins; principles are qualitatively } \\
\text { postulated and observed; focus is on new discovery rather than applications }\end{array}$ \\
\hline TRL 2 & $\begin{array}{c}\text { Applied research: initial practical applications are identified; potential of material } \\
\text { or process to solve a problem, satisfy a need, or find application is confirmed }\end{array}$ \\
\hline TRL 3 & $\begin{array}{c}\text { Critical function or proof of concept established: applied research advances and } \\
\text { early stage development begins; studies and laboratory measurements validate } \\
\text { analytical predictions of separate elements of the technology }\end{array}$ \\
\hline TRL 4 & $\begin{array}{c}\text { Lab testing/validation of alpha prototype component/process: design, } \\
\text { development, and lab testing of components/processes; results provide evidence that } \\
\text { performance targets may be attainable based on projected or modeled systems (alfa } \\
\text { test) }\end{array}$ \\
\hline TRL 5 & $\begin{array}{c}\text { Laboratory testing of integrated/semi-integrated system: system component } \\
\text { and/or process validation is achieved in a relevant environment }\end{array}$ \\
\hline TRL 6 & $\begin{array}{r}\text { Prototype system verified: system/process prototype demonstration in an } \\
\text { operational environment (beta test) }\end{array}$ \\
\hline TRL 7 & $\begin{array}{c}\text { Integrated pilot system demonstrated: system/process prototype demonstration in } \\
\text { an operational environment (integrated pilot system level) }\end{array}$
\end{tabular}


Table 1. Continued.

\begin{tabular}{|c|c|}
\hline TRL 8 & $\begin{array}{c}\text { System incorporated in commercial design: actual system/process completed and } \\
\text { qualified through test and demonstration (pre-commercial demonstration) }\end{array}$ \\
\hline TRL 9 & $\begin{array}{c}\text { System proven and ready for full commercial deployment: actual system proven } \\
\text { through successful operations in operating environment, and ready for full } \\
\text { commercial deployment }\end{array}$ \\
\hline
\end{tabular}

Note. Based on data from [3].

The main research activity ends with obtaining initial results and early insights. Time lag for turning into a viable product and having to invest significant resources to pursue the required development may be regarded as major barriers and discouraging factors contributing to business establishments' reluctance to work with higher education institutions, which may diminish investment incentives and limit opportunities for funding.

This pattern is especially the case in highly knowledge-intensive sectors, where specific market and statutory conditions further raise the market entry threshold in terms of both timeframes and resources needed. This gap limits the capacity of academic teams to access external funding. Such limitations tend to impede cooperation between university researchers and investors, which translates into the need for intermediaries to help bridge the communication gap by providing dedicated facilities and consulting assistance [2].

Recent years have seen a growing interest in the development and use of the following two intercomplementary financial/consulting instruments intended to facilitate the maturation and commercialization of university-generated technologies: (1) proof of concept testing by expert centers and (2) investment from seed funds associated with university incubators and accelerators. However, activating such instruments of funding, their optimal use, and their ultimate efficiency is a current barrier to their use in Russia. Even in the USA and the EU the interest in improving the overall technology transfer system remains high [4].

A possible variant of the conceptual and structural/logical model for the mechanism of the innovation commercialization in higher education institutions is the establishment and operation of proof of concept centers (PoCCs). Intrinsically, PoCCs are an organizational innovation intended to deal with issues relating to the commercialization of university-based research and support the development of university startups.

The PoCC enables researchers and inventors to assess the commercial potential of their inventions at an early stage and test their prototypes for commercial usability in the future. Proof of concept makes it easier for inventors to obtain funding for their further development of the product from external (e.g. angel or venture) investors, as well as receiving consumers' feedback at an early stage and get confirmation of the viability of the technology $[5 ; 6]$.

This work will be carried out throughout the innovation's lifecycle - from the initial idea through to implementation via a methodology such as TPRL (Technology Project Readiness Levels), a tool developed in Russia with assistance from technology brokers to assess the readiness degree of a technological product for using by business. The tool combines elements of two internationally recognized methodologies - TRL (Technology Readiness Levels), used to assess the maturity of technology readiness, and MRL (Manufacturing Readiness Levels), used to assess the maturity of manufacturing readiness [7].

At each stage of the research process, jointly with the technology transfer office (TTO), the PoCC is there to provide all kinds of assistance, including attracting external partners and investors, ensuring marketing and legal support, and attracting orders for the technologies. This can also be achieved by the provision of initial funding to researchers in the early stages of the process. This implies that the research must be in line with the needs of the market, focusing on two-way communication with the market right from the earliest stages [5].

It is important that within the PoCC's partner environment there be sustainable links for interaction, including patent agents, product development consultants, technological brokers, business angels (angel investors), venture capitalists, and interested firms - with which the 
higher education institution could cooperate. This component is necessary in that potential partners representing industry should be able to invest in risky or unproven technologies with an awareness that there is an external supporting infrastructure out there to support further development and commercialization.

An upside to initial funding (seed investment) via the PoCC is that there is double confirmation of the practical realization of research outcomes: (1) obtaining an informed expert judgment on the idea's viability, market interest in it and professional consulting assistance on intellectual property rights; and (2) the PoCC, which is commercially interested in its own investment, is there to provide initial financial support to help university researchers demonstrate the feasibility of their discoveries. The end objective is to take the technology to a level of possibility to be licensed for external industrial partners or setting up a spin-off company for attracting investors in subsequent stages of development [6;8].

While being primarily a mechanism for support and guidance in research commercialization intended to reduce the technological uncertainty of research projects, the PoCC is far superior in feature set and capabilities to the TTO, which steps in the final stages of the transfer and commercialization process. Therefore, in terms of the structural model, it is logical to incorporate the TTO's functions into the PoCC's extended structure.

A higher education institution may support research projects in various ways, including assistance from third parties, like business incubators or accelerators (Table 2).

Table 2. Model for Support for the Commercialization of University R\&D Outputs at Different Technology Readiness Levels (TRLs).

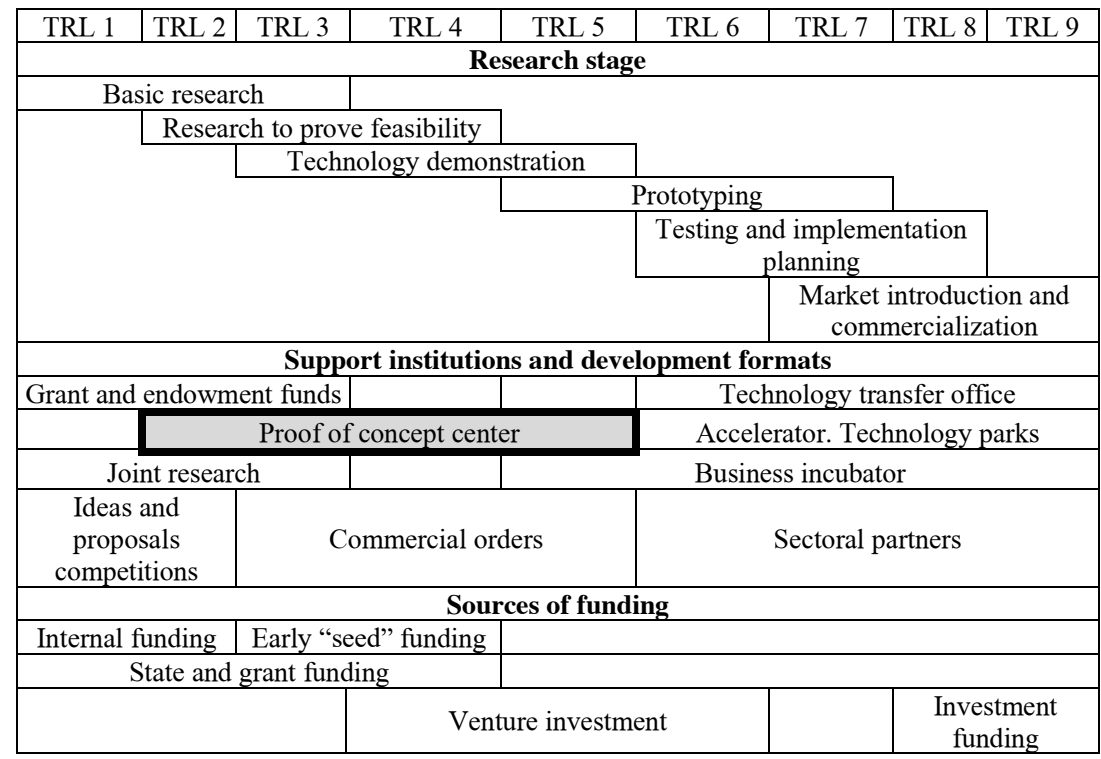

Note. Produced by the authors based on their research findings.

Transforming research ideas and outcomes into commercial products or services requires special competencies and skills, as well as special infrastructural and ecosystemic conditions. The proposed unit may serve as an effective decision-making tool for assessing potential cooperation in the researches with industrial partners. At a practical level, the developed unit may help assess the probability of success and potential issues for specific projects.

It may be worth using a system that can take account of the following levels of analysis:

- type and format of the knowledge being transferred; 
- internal processes, participating persons, an organization's units, and an organization's resources (to be applied to all the interested parties involved in the cooperation);

- internal and external policy of the parties involved in the technology transfer process;

- level of business intercorporate culture (to ensure long-term sustainable cooperation).

Thus, instead of directly activating and managing their own funding programs, small universities may find it more rational to explore the possibility of developing network cooperation, at a regional level, with other universities and/or regional authorities with a view to forming linked projects and technologies between institutions, reducing operating costs, and attracting a more professional workforce [9; 10]. Essentially, a general model for innovation commercialization may be illustrated as the next succession of processes and participants (Figure 1.)

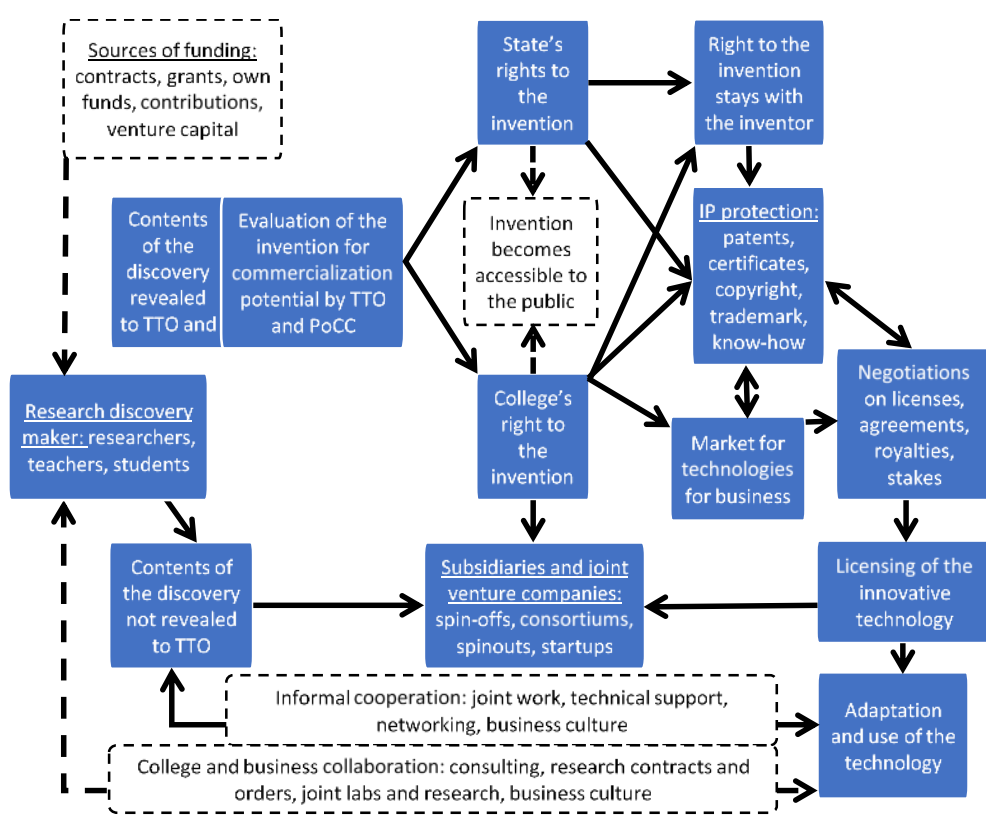

Fig. 1. Expanded model for the commercialization and transfer of university-generated technologies. Produced by the authors based on their research findings.

\section{Conclusions}

Firstly, from a standpoint of managerial transformations and changes to policy, the format of proof of concept centers has importance as an instrument for boosting the market attractiveness and investment readiness of designs generated in university research. Available PoCC is becoming a crucial component in the technology transfer ecosystem today.

Secondly, given the importance of these instruments for a wide range of organizations, they do not necessarily have to be established and managed by a single university.

Thirdly, there is a need for highly qualified human capital, especially by industry requirements, typical competencies of transfer technology office employees, technology brokers, etc., and in today's embodiment of scientific/research and production/entrepreneurial activity in higher education institutions. 
In addition to these general conditions, there are a number of critical factors governing the success of PoCC programs, such as the following:

- the program being funded both from funds provided by third parties and partners and from the university's own funds;

- instruments of additional support being available for the development of projects launched with participation from the higher education institution;

- a flexible, individualized and liberal approach to the interaction of the higher education institution with the being created establishment and third-party professionals.

A better understanding of the key factors influencing the creation of measures related to funding in universities will be beneficial for universities' policy and organizational practice decisions and will help guide the choice of government policy, which potentially should help create more favorable conditions for the successful use of research outputs [11].

\section{References}

1. G. E. Evans, Intellectual property commercialization: Policy options and practical instr uments (2011)

2. F. Munari, M. Sobrero, L. Toschi, Bridging the University Funding Gap: Determinants and Consequences of University Seed Funds and Proof-of-Concept Programs in Europe (2015)

3. The TRL Scale as a Research \& Innovation Policy Tool, EARTO Recommendations (2014)

4. A. N. Link, C. S. Hayter, T. J. of Techn Tr, 40(1) (2015)

5. C. A. Gulbranson, D. B. Audretsch, T. J. of Techn Tr, 33(3) (2008)

6. F. Munari, M. Sobrero, L. Toschi, Techn Anal \& Strat Manag, 29 (2017)

7. A. Petrov, A. Sartori, A. Filimonov, Econ of Sc, 2(4) (2016)

8. J. Wonglimpiyarat, Technology Financing and Commercialization (2015)

9. K. Vishnevsky, J. Calof, D. Meissner, Futures Thinking and Organizational Policy: Case Studies for Managing Rapid Change in Technology, Globalization and Workforce Diversity (2019)

10. M. Gershman, T. Zinina, M. Romanov et al., Innovation Development Programmes of Russian State-Owned Companies: Interim Results and Priorities (2015)

11. Methodology for the Development of National Intellectual Property Strategies (2016) 